Kragujevac Journal of Mathematics

Volume 42(3) (2018), Pages 419-430.

\title{
GENERALIZED SUZUKI TYPE Z-CONTRACTION IN COMPLETE METRIC SPACES
}

\begin{abstract}
A. PADCHAROEN ${ }^{1,2}$, P. KUMAM ${ }^{1,2}$, P. SAIPARA ${ }^{1,2}$, AND P. CHAIPUNYA $^{1,2}$
ABSTRACT. In this paper, we introduce the notion of Suzuki type Z-contraction and study the corresponding fixed point property. This kind of contraction generalizes the Banach contraction and unifies several known type of nonlinear contractions. We consider a nonlinear operator satisfying a nonlinear contraction in a metric space and prove fixed point results. As an application, we apply our result to show the solvability of nonlinear Hammerstein integral equations.
\end{abstract}

\section{Introduction AND Preliminaries}

One of the attractive research subjects in the fixed point theory is the investigation of the existence and uniqueness of fixed point of various operators in the setting of metric space. The classical results of Banach [2] and Suzuki [8] have inspired many scientists and hence there is a wide literature available for interested readers, see for instance $[1,3,5,7,9-11]$. We give some details on the notions and ideas used in this study.

Recall the following result on metric space which is introduced in 2009 by Suzuki. He also proved the following generalized Banach contraction principle in compact metric spaces as follows.

Theorem 1.1. [8] Let $(X, d)$ be a compact metric space and $F: X \rightarrow X$ be a mapping. Assume that

$$
\frac{1}{2} d(x, F x)<d(x, y) \Rightarrow d(F x, F y)<d(x, y),
$$

Key words and phrases. Suzuki type Z-contraction, fixed point, metric space, nonlinear Hammerstein integral equations.

2010 Mathematics Subject Classification. Primary: 47H09. Secondary: 47H10, 37C25.

*Corresponding author: poom.kum@kmutt.ac.th (P. Kumam)

Received: June 05, 2016.

Accepted: April 12, 2017. 
for all distinct $x, y \in X$. Then $F$ has a unique fixed point in $X$.

In 2015, Khojasteh et al. [4] introduced the notion of Z-contraction which generalizes the Banach contraction. The notion of Z-contraction is given as follows.

Definition 1.1. [4] Let $\zeta:[0,+\infty) \times[0,+\infty) \rightarrow \mathbb{R}$ be a mapping, then $\zeta$ is called a simulation function if it satisfies the following conditions:

$\zeta(i) \zeta(0,0)=0$

$\zeta(i i) \zeta(t, s)<s-t$, for all $t, s>0$;

$\zeta($ iii $)$ if $\left\{t_{n}\right\},\left\{s_{n}\right\}$ are sequences in $(0,+\infty)$ such that $\lim _{n \rightarrow+\infty} t_{n}=\lim _{n \rightarrow+\infty} s_{n}>0$ then

$$
\limsup _{n \rightarrow+\infty} \zeta\left(t_{n}, s_{n}\right)<0 .
$$

We denote the set of all simulation functions by $z$.

The following functions $\zeta:[0,+\infty) \times[0,+\infty) \rightarrow \mathbb{R}$ belong to $z$.

Definition 1.2. [4] Let $(X, d)$ be a metric space, $F: X \rightarrow X$ a mapping and $\zeta \in z$. Then $F$ is called a $Z$-contraction with respect to $\zeta$, if the following condition is satisfied

$$
\zeta(d(F x, F y), d(x, y)) \geq 0, \text { for all } x, y \in X .
$$

Recently, Kumam et al. [6] introduce the motion Suzuki type z-contraction as follows.

Definition 1.3. [6] Let $(X, d)$ be a metric space, $F: X \rightarrow X$ a mapping and $\zeta \in z$. Then $F$ is called a Suzuki type $z$-contraction with respect to $\zeta$, if the following condition is satisfied

$$
\frac{1}{2} d(x, F x)<d(x, y) \Rightarrow \zeta(d(F x, F y), d(x, y)) \geq 0,
$$

for all distinct $x, y \in X$.

Remark 1.1. [6] It is clear from the definition of simulation function that $\zeta(t, s)<0$, for all $t \geq s>0$. Therefore if $F$ is a Suzuki type $z$-contraction with respect to $\zeta$, then

for all distinct $x, y \in X$.

$$
\frac{1}{2} d(x, F x)<d(x, y) \Rightarrow d(F x, F y)<d(x, y),
$$

Theorem 1.2. [6] Let $(X, d)$ be a metric space and $F: X \rightarrow X$ be a Suzuki type $z$-contraction with respect to $\zeta \in Z$. Then $F$ has at most one fixed point.

Remark 1.2. [4] Every Z-contraction is contractive and hence Banach contraction.

Theorem 1.3. [2] Let $(X, d)$ be a complete metric space. Then every contraction mapping has a unique fixed point. It is known as Banach contraction principle.

Motivated by the above results, we introduce the notion Generalized Suzuki type Z-contraction and prove the corresponding fixed point theorem. Our fixed point result is then applied to guarantee the existence of solution of nonlinear Hammerstein integral equations. 


\section{MAin Results}

We introduce the concept of generalized Suzuki type Z-contraction on metric spaces with the following auxiliary definition. Let $X \neq \emptyset, F: X \rightarrow X, x_{0} \in X$ and $x_{n}=F x_{n-1}$, for all $n \in \mathbb{N}$. Then $\left\{x_{n}\right\}$ is called sequence of Picard of initial point at $x_{0}$.

Definition 2.1. Let $(X, d)$ be a metric space and $F: X \rightarrow X$ be a mapping and $\zeta \in z$. Then $F$ is called generalized Suzuki type $Z$-contraction with respect to $\zeta$ if the following condition is satisfied

$$
\frac{1}{2} d(x, F x)<d(x, y) \Rightarrow \zeta(d(F x, F y), M(x, y)) \geq 0,
$$

for all distinct $x, y \in X$, where

$$
M(x, y)=\max \left\{d(x, y), d(x, F x), d(y, F y), \frac{d(x, F y)+d(y, F x)}{2}\right\} .
$$

Remark 2.1. It is clear from the definition of simulation function that $\zeta(t, s)<0$, for all $t \geq s>0$. Therefore $F$ is a generalized Suzuki type $Z$-contraction with respect to $\zeta$, then

for all distinct $x, y \in X$.

$$
\frac{1}{2} d(x, F x)<d(x, y) \Rightarrow d(F x, F y)<M(x, y),
$$

Lemma 2.1. Every generalized Suzuki type Z-contraction with respect to $\zeta$ on metric space has at most one fixed point.

Proof. Suppose that $w$ and $z$ be two fixed points of $F$, which is a generalized Suzuki type Z-contraction self-mappings of a metric space $(X, d)$. Since $0=\frac{1}{2} d(w, F w)<$ $d(w, z)$, then by applying (2.1), we obtain that

$$
0 \leq \zeta(d(F w, F z), M(w, z))
$$

where

$$
M(w, z)=\max \left\{d(w, z), d(w, F w), d(z, F z), \frac{d(w, F z)+d(z, F w)}{2}\right\}=d(w, z) .
$$

This together with (2.2) shows that

$$
0 \leq \zeta(d(F w, F z), M(w, z))=\zeta(d(w, z), d(w, z)) .
$$

This is a contradiction. Thus, we have $w=z$.

Theorem 2.1. Let $(X, d)$ be a metric space, $F$ is a generalized Suzuki type Zcontraction with respect to $\zeta$. Let $\left\{x_{n}\right\}$ be a sequence of Picard of initial point at $x_{0} \in X$. Then

$$
\lim _{n \rightarrow+\infty} d\left(x_{n}, x_{n+1}\right)=0 .
$$


Proof. Take $x_{0}=x \in X$ and let $\left\{x_{n}\right\}$ be the Picard sequence, that is, $x_{n}=F x_{n-1}$, for all $n \in \mathbb{N}$. If there exists $n \in \mathbb{N}$ such that $d\left(x_{n}, F x_{n}\right)=0$ then $x=x_{n}$ becomes a fixed point of $F$, which completes the proof. So, in the rest of the proof, we suppose that

$$
0<d\left(x_{n}, F x_{n}\right), \text { for all } n \in \mathbb{N} \text {. }
$$

Hence, we have

$$
\frac{1}{2} d\left(x_{n}, F x_{n}\right)<d\left(x_{n}, F x_{n}\right)=d\left(x_{n}, x_{n+1}\right) .
$$

Since $F$ is a generalized Suzuki type $Z$-contraction, we have

$$
0 \leq \zeta\left(d\left(F x_{n}, F^{2} x_{n}\right), M\left(x_{n}, F x_{n}\right)\right)=\zeta\left(d\left(F x_{n}, F x_{n+1}\right), M\left(x_{n}, x_{n+1}\right)\right) .
$$

Then

$$
\begin{aligned}
& M\left(x_{n}, x_{n+1}\right) \\
= & \max \left\{d\left(x_{n}, x_{n+1}\right), d\left(x_{n}, x_{n+1}\right), d\left(x_{n+1}, x_{n+2}\right), \frac{d\left(x_{n}, x_{n+2}\right)+d\left(x_{n+1}, x_{n+1}\right)}{2}\right\} \\
= & \max \left\{d\left(x_{n}, x_{n+1}\right), d\left(x_{n+1}, x_{n+2}\right), \frac{d\left(x_{n}, x_{n+2}\right)}{2}\right\} .
\end{aligned}
$$

The triangle inequality yields

$$
\frac{d\left(x_{n}, x_{n+2}\right)}{2} \leq \max \left\{d\left(x_{n}, x_{n+1}\right), d\left(x_{n+1}, x_{n+2}\right)\right\} .
$$

Therefore,

$$
M\left(x_{n}, x_{n+1}\right)=\max \left\{d\left(x_{n}, x_{n+1}\right), d\left(x_{n+1}, x_{n+2}\right)\right\},
$$

from (2.1), we get that

$$
\begin{aligned}
0 & \leq \zeta\left(d\left(F x_{n}, F x_{n+1}\right), M\left(x_{n}, x_{n+1}\right)\right) \\
& =\zeta\left(d\left(x_{n+1}, x_{n+2}\right), \max \left\{d\left(x_{n}, x_{n+1}\right), d\left(x_{n+1}, x_{n+2}\right)\right\}\right) \\
& <\max \left\{d\left(x_{n}, x_{n+1}\right), d\left(x_{n+1}, x_{n+2}\right)\right\}-d\left(x_{n+1}, x_{n+2}\right) .
\end{aligned}
$$

The inequality (2.3) shows that

$$
M\left(x_{n}, x_{n+1}\right)=d\left(x_{n}, x_{n+1}\right) \text {, for all } n \in \mathbb{N},
$$

which implies that the sequence $\left\{d\left(x_{n}, x_{n+1}\right)\right\}$ is a monotonically decreasing sequence of non-negative reals. So there is some $r \geq 0$ such that

$$
\lim _{n \rightarrow+\infty} d\left(x_{n}, x_{n+1}\right)=r .
$$

If $r>0$ then since $F$ is a generalized Suzuki type $z$-contraction with respect to $\zeta \in z$ therefore by $\zeta(i i i)$, we have

$$
0 \leq \limsup _{n \rightarrow+\infty} \zeta\left(d\left(x_{n+1}, x_{n+2}\right), d\left(x_{n}, x_{n+1}\right)\right)<0 .
$$

This is a contradiction. Then we conclude that $r=0$, that is, $\lim _{n \rightarrow+\infty} d\left(x_{n}, x_{n+1}\right)=$ 0 . 
Theorem 2.2. Let $(X, d)$ be a metric space, $F$ is a generalized Suzuki type Zcontraction with respect to $\zeta$. Then the sequence $\left\{x_{n}\right\}$ is bounded.

Proof. Suppose that $\left\{x_{n}\right\}$ is not bounded sequence. Then there a subsequence $\left\{x_{n_{k}}\right\}$ of $\left\{x_{n}\right\}$ such that $n_{1}=1$ and for each $k \in \mathbb{N}, n_{k+1}$ is the minimum integer such that

$$
d\left(x_{n_{k+1}}, x_{n_{k}}\right)>1
$$

and

$$
d\left(x_{m}, x_{n_{k}}\right) \leq 1 \text { for } n_{k} \leq m \leq n_{k+1}-1
$$

Thus, by the triangle inequality, we get

$$
\begin{aligned}
1<d\left(x_{n_{k+1}}, x_{n_{k}}\right) & \leq d\left(x_{n_{k+1}}, x_{n_{k+1}-1}\right)+d\left(x_{n_{k+1}-1}, x_{n_{k}}\right) \\
& \leq d\left(x_{n_{k+1}}, x_{n_{k+1}-1}\right)+1 .
\end{aligned}
$$

Letting $k \rightarrow+\infty$ and by using Theorem 2.1 , we obtain

$$
\lim _{k \rightarrow+\infty} d\left(x_{n_{k+1}}, x_{n_{k}}\right)=1
$$

Since

$$
\frac{1}{2} d\left(x_{n_{k+1}-1}, F x_{n_{k}-1}\right)=\frac{1}{2} d\left(x_{n_{k+1}-1}, x_{n_{k}}\right)<d\left(x_{n_{k+1}-1}, x_{n_{k}}\right),
$$

by generalized Suzuki type $Z$-contraction with respect to $\zeta$, we get that

$$
d\left(x_{n_{k+1}}, x_{n_{k}}\right) \leq M\left(x_{n_{k+1}-1}, x_{n_{k}-1}\right) .
$$

Now,

$$
\begin{aligned}
1 & <d\left(x_{n_{k+1}}, x_{n_{k}}\right) \leq M\left(x_{n_{k+1}-1}, x_{n_{k}-1}\right) \\
& =\max \left\{\begin{array}{c}
d\left(x_{n_{k+1}-1}, x_{n_{k}-1}\right), d\left(x_{n_{k+1}-1}, x_{n_{k+1}}\right), d\left(x_{n_{k}-1}, x_{n_{k}}\right), \\
\frac{d\left(x_{n_{k+1}-1}, x_{n_{k}}\right)+d\left(x_{n_{k}-1}, x_{n_{k+1}}\right)}{2}
\end{array}\right\} \\
& =\max \left\{\begin{array}{c}
d\left(x_{n_{k+1}-1}, x_{n_{k}}\right)+d\left(x_{n_{k}}, x_{n_{k}-1}\right), d\left(x_{n_{k+1}-1}, x_{n_{k+1}}\right), d\left(x_{n_{k}-1}, x_{n_{k}}\right), \\
\frac{d\left(x_{n_{k+1}-1}, x_{n_{k}}\right)+d\left(x_{n_{k}-1}, x_{n_{k+1}}\right)}{2}
\end{array}\right\} \\
& \leq \max \left\{\begin{array}{c}
\left.1+d\left(x_{n_{k}}, x_{n_{k}-1}\right), d\left(x_{n_{k+1}-1}, x_{n_{k+1}}\right), d\left(x_{n_{k}-1}, x_{n_{k}}\right), \frac{1+d\left(x_{n_{k}-1}, x_{n_{k+1}}\right)}{2}\right\} \\
\leq
\end{array}\right\} .
\end{aligned}
$$

Letting $k \rightarrow+\infty$, we obtain

$$
1 \leq \lim _{k \rightarrow+\infty} M\left(x_{n_{k+1}-1}, x_{n_{k}-1}\right) \leq 1,
$$


that is,

$$
\lim _{k \rightarrow+\infty} M\left(x_{n_{k+1}-1}, x_{n_{k}-1}\right)=1 .
$$

Further, since $\frac{1}{2} d\left(x_{n_{k+1}-1}, x_{n_{k}-1}\right)<d\left(x_{n_{k+1}-1}, x_{n_{k}-1}\right)$. Therefore, $F$ is a generalized Suzuki type $z$-contraction with respect to $\zeta$, we obtain

$$
\begin{aligned}
0 & \leq \zeta\left(d\left(F x_{n_{k+1}-1}, F x_{n_{k}-1}\right), M\left(x_{n_{k+1}-1}, x_{n_{k}-1}\right)\right) \\
& \leq \limsup _{k \rightarrow+\infty} \zeta\left(d\left(x_{n_{k+1}}, x_{n_{k}}\right), M\left(x_{n_{k+1}-1}, x_{n_{k}-1}\right)\right)<0 .
\end{aligned}
$$

This is a contradiction. Hence, $\left\{x_{n}\right\}$ is bounded.

Theorem 2.3. Let $(X, d)$ be a metric space, $F$ is a generalized Suzuki type Zcontraction with respect to $\zeta$. Then the sequence $\left\{x_{n}\right\}$ is a Cauchy sequence.

Proof. Let $C_{n}=\sup \left\{d\left(x_{i}, x_{j}\right): i, j \geq n\right\}, n \in \mathbb{N}$. From Theorem 2.2, we know that $C_{n}<+\infty$ for every $n \in \mathbb{N}$. Since $C_{n}$ is a positive monotonically decreasing sequence, there exists $C \geq 0$ such that $\lim _{n \rightarrow+\infty} C_{n}=C$. We will show that $C=0$. If $C>0$ then by the definition of $C_{n}$, for every $k \in \mathbb{N}$, there exists $n_{k}, m_{k}$ such that $m_{k}>n_{k} \geq k$ and

Therefore,

$$
C_{k}-\frac{1}{k}<d\left(x_{m_{k}}, x_{n_{k}}\right) \leq C_{k}
$$

$$
\lim _{k \rightarrow+\infty} d\left(x_{m_{k}}, x_{n_{k}}\right)=C
$$

Moreover, by

$$
d\left(x_{m_{k}}, x_{n_{k}}\right) \leq d\left(x_{m_{k}}, x_{m_{k}-1}\right)+d\left(x_{m_{k}-1}, x_{n_{k}-1}\right)+d\left(x_{n_{k}-1}, x_{n_{k}}\right)
$$

and

$$
d\left(x_{m_{k}-1}, x_{n_{k}-1}\right) \leq d\left(x_{m_{k}-1}, x_{m_{k}}\right)+d\left(x_{m_{k}}, x_{n_{k}}\right)+d\left(x_{n_{k}}, x_{n_{k}-1}\right) .
$$

Letting $k \rightarrow+\infty$, using Theorem 2.1 and equation (2.5), we get

$$
\lim _{k \rightarrow+\infty} d\left(x_{m_{k}-1}, x_{n_{k}-1}\right)=C .
$$

From Theorem 2.1 and (2.6), we obtain that $\frac{1}{2} d\left(x_{m_{k}-1}, F x_{m_{k}-1}\right)<\frac{1}{2} d\left(x_{m_{k}-1}, x_{n_{k}-1}\right)$ $<d\left(x_{m_{k}-1}, x_{n_{k}-1}\right)$. By $F$ is a generalized Suzuki type $z$-contraction with respect to $\zeta$, we have $0 \leq \zeta\left(d\left(F x_{m_{k}-1}, F x_{n_{k}-1}\right), M\left(x_{m_{k}-1}, x_{n_{k}-1}\right)\right)$. It follows from condition $\zeta($ ii $)$ we get

$$
\begin{aligned}
& d\left(x_{m_{k}}, x_{n_{k}}\right)=d\left(F x_{m_{k}-1}, F x_{n_{k}-1}\right)<M\left(x_{m_{k}-1}, x_{n_{k}-1}\right) \\
= & \max \left\{\begin{array}{c}
d\left(x_{m_{k}-1}, x_{n_{k}-1}\right), d\left(x_{m_{k}-1}, x_{m_{k}}\right), d\left(x_{n_{k}-1}, x_{n_{k}}\right), \\
\frac{d\left(x_{m_{k}-1}, x_{n_{k}}\right)+d\left(x_{m_{k}}, x_{n_{k}-1}\right)}{2}
\end{array}\right\} \\
= & \max \left\{\begin{array}{c}
d\left(x_{m_{k}-1}, x_{n_{k}-1}\right), d\left(x_{m_{k}-1}, x_{m_{k}}\right), d\left(x_{n_{k}-1}, x_{n_{k}}\right), \\
\frac{d\left(x_{m_{k}-1}, x_{m_{k}}\right)+d\left(x_{m_{k}}, x_{n_{k}}\right)+d\left(x_{m_{k}}, x_{n_{k}}\right)+d\left(x_{n_{k}}, x_{n_{k}-1}\right)}{2}
\end{array}\right\} .
\end{aligned}
$$


Letting $k \rightarrow+\infty$, using Theorem 2.1 and equation (2.5), we get

$$
\lim _{k \rightarrow+\infty} M\left(x_{m_{k}-1}, x_{n_{k}-1}\right)=C .
$$

By (2.6), (2.7) and the condition $\zeta($ iii $)$, we get

$$
0 \leq \limsup _{k \rightarrow+\infty} \zeta\left(d\left(x_{m_{k}}, x_{n_{k}}\right), M\left(x_{m_{k}-1}, x_{n_{k}-1}\right)\right)<0 .
$$

This is a contradiction. Hence, $C=0$. This is $\left\{x_{n}\right\}$ is a Cauchy sequence.

Theorem 2.4. Let $(X, d)$ be a complete metric space, $F$ is a generalized Suzuki type $Z$-contraction with respect to $\zeta$. Then $F$ has fixed point.

Proof. By Theorem 2.3, $\left\{x_{n}\right\}$ is a Cauchy sequence and $X$ is complete there exists $w \in X$ such that

$$
\lim _{n \rightarrow+\infty} x_{n}=w
$$

Now, we show that $w$ is a fixed point of $F$. We claim that

$$
\frac{1}{2} d\left(x_{n}, F x_{n}\right)<d\left(x_{n}, w\right) \text { or } \frac{1}{2} d\left(x_{n+1}, F x_{n+1}\right)<d\left(x_{n+1}, w\right), \text { for all } n \in \mathbb{N} .
$$

This is,

(2.9) $\frac{1}{2} d\left(x_{n}, F x_{n}\right)<d\left(x_{n}, w\right)$ or $\frac{1}{2} d\left(F x_{n}, F^{2} x_{n}\right)<d\left(F x_{n}, w\right)$, for all $n \in \mathbb{N}$.

It follows from $(2.9)$.

Let $(I):=\frac{1}{2} d\left(x_{n}, F x_{n}\right)<d\left(x_{n}, w\right)$ and $(I I):=\frac{1}{2} d\left(F x_{n}, F^{2} x_{n}\right)<d\left(F x_{n}, w\right)$. Assume that there exists $m \in \mathbb{N}$ such that

$$
\frac{1}{2} d\left(x_{m}, F x_{m}\right) \geq d\left(x_{m}, w\right) \text { and } \frac{1}{2} d\left(F x_{m}, F^{2} x_{m}\right) \geq d\left(F x_{m}, w\right) .
$$

Hence,

$$
2 d\left(x_{m}, w\right) \leq d\left(x_{m}, F x_{m}\right) \leq d\left(x_{m}, w\right)+d\left(w, F x_{m}\right) .
$$

This implies that

$$
d\left(x_{m}, w\right) \leq d\left(w, F x_{m}\right)
$$

Form (2.4) and (2.11), we have

$$
\begin{aligned}
d\left(F x_{m}, F^{2} x_{m}\right) & <d\left(x_{m}, F x_{m}\right) \leq d\left(x_{m}, w\right)+d\left(w, F x_{m}\right) \\
& <2 d\left(w, F x_{m}\right) .
\end{aligned}
$$

It follows, from (2.10) and (2.12), that $d\left(F x_{m}, F^{2} x_{m}\right)<d\left(F x_{m}, F^{2} x_{m}\right)$. This is a contradiction. Thus, (2.9) holds. If part $(I)$ of $(2.9)$ is true, by $F$ is a generalized Suzuki type $Z$-contraction with respect to $\zeta$, we have

$$
0 \leq \zeta\left(d\left(F x_{n}, F w\right), M\left(x_{n}, w\right)\right) .
$$


It follows from condition $\zeta(i i)$ we get

$$
\begin{aligned}
d\left(F x_{n}, F w\right) & <M\left(x_{n}, w\right) \\
& =\max \left\{d\left(x_{n}, w\right), d\left(x_{n}, F x_{n}\right), d(w, F w), \frac{d\left(x_{n}, F w\right)+d\left(w, F x_{n}\right)}{2}\right\} .
\end{aligned}
$$

Letting $n \rightarrow+\infty$ and by using (2.8), we obtain

$$
\lim _{n \rightarrow+\infty} M\left(x_{n}, w\right)=d(w, F w) .
$$

By (2.13), (2.14) and condition $\zeta($ iii $)$ we have for every $n \in \mathbb{N}$,

$$
\begin{aligned}
0 & \leq \zeta\left(d\left(F x_{n}, F w\right), M\left(x_{n}, w\right)\right) \\
& \leq \limsup _{n \rightarrow+\infty} \zeta\left(d\left(F x_{n}, F w\right), M\left(x_{n}, w\right)\right) \\
& \leq \limsup _{n \rightarrow+\infty}(d(w, F w)-d(w, F w)) .
\end{aligned}
$$

According to the property $\zeta($ iii $)$ from Definition 1.1, since the both sequences $d\left(F x_{n}, F w\right), M\left(x_{n}, w\right)$ converge to the $d(w, F w)>0$ (by assumption) it is clear that $\lim \sup _{n \rightarrow+\infty} \zeta\left(d\left(F x_{n}, F w\right), M\left(x_{n}, w\right)\right)<0$. So, the mention inequality is redundant. This is a contradiction. Hence, $w=F w$, i.e., $w$ is a fixed point of $F$. If part $(I I)$ of (2.9) is true, by $F$ is a generalized Suzuki type $z$-contraction with respect to $\zeta$, we have

$$
0 \leq \zeta\left(d\left(F^{2} x_{n}, F w\right), M\left(F x_{n}, w\right)\right) .
$$

It follows from condition $\zeta(i i)$, we get

$$
\begin{aligned}
d\left(F^{2} x_{n}, F w\right) & <M\left(F x_{n}, w\right) \\
& =\max \left\{\begin{array}{c}
d\left(F x_{n}, w\right), d\left(F x_{n}, F^{2} x_{n}\right), d(w, F w), \\
\frac{d\left(F x_{n}, F w\right)+d\left(w, F^{2} x_{n}\right)}{2}
\end{array}\right\} \\
& =\max \left\{\begin{array}{c}
d\left(F x_{n}, w\right), d\left(F x_{n}, F^{2} x_{n}\right), d(w, F w), \\
\frac{d\left(F x_{n}, F w\right)+d(w, F w)+d\left(F w, F^{2} x_{n}\right)}{2}
\end{array}\right\} .
\end{aligned}
$$

Letting $n \rightarrow+\infty$ and by using (2.8), we obtain

$$
\lim _{n \rightarrow+\infty} M\left(F x_{n}, w\right)=d(w, F w) .
$$

By (2.15), (2.16) and condition $\zeta($ iii $)$ we have for every $n \in \mathbb{N}$,

$$
\begin{aligned}
0 & \leq \zeta\left(d\left(F^{2} x_{n}, F w\right), M\left(F x_{n}, w\right)\right) \\
& \leq \limsup _{n \rightarrow+\infty} \zeta\left(d\left(F^{2} x_{n}, F w\right), M\left(F x_{n}, w\right)\right) \\
& \leq \limsup _{n \rightarrow+\infty}(d(w, F w)-d(w, F w)) .
\end{aligned}
$$

According to the property $\zeta(i i i)$ from Definition 1.1, since the both sequences $d\left(F^{2} x_{n}, F w\right), M\left(F x_{n}, w\right)$ converge to the $d(w, F w)>0$ (by assumption) it is clear 
that $\limsup _{n \rightarrow+\infty} \zeta\left(d\left(F^{2} x_{n}, F w\right), M\left(F x_{n}, w\right)\right)<0$. So, the mention inequality is redundant. This is a contradiction. Hence, $w=F w$, i.e., $w$ is a fixed point of $F$. Uniqueness of fixed point follows from Lemma 2.1.

Example 2.1. Let $X=[0,1]$ and $d$ is a usual metric on $X$. Define a mapping $F: X \rightarrow X$ by

for all $x \in X$, then

$$
F x= \begin{cases}\frac{4}{9}, & \text { if } x \in\left[0, \frac{4}{5}\right), \\ \frac{1}{9}, & \text { if } x \in\left[\frac{4}{5}, 1\right]\end{cases}
$$

$$
d(F x, F y)<M(x, y) .
$$

Therefore, we have

$$
\zeta(d(F x, F y), M(x, y)) \geq 0,
$$

for all distinct $x, y \in X$. Hence, $F$ is a generalized Suzuki type Z-contraction with respect to $\zeta$. Thus, all the condition of Theorem 2.4 are satisfied and $F$ has a unique fixed point $w=\frac{4}{9} \in X$. Since $F$ is not continuous, then it is not Z-contraction and so not contractive.

Corollary 2.1. Let $(X, d)$ be a complete metric space, $F: X \rightarrow X$ be a mapping such that there exists $k \in(0,1)$ verifying

$$
d(F x, F y)<k M(x, y), \text { for all distinct } x, y \in X,
$$

where

$$
M(x, y)=\max \left\{d(x, y), d(x, F x), d(y, F y), \frac{d(x, F y)+d(y, F x)}{2}\right\} .
$$

Then $F$ has a fixed point.

\section{Application}

In this section, we present an application of Theorem to guarantee the existence and uniqueness problem of solutions for some kind of nonlinear Hammerstein integral equations.

We consider nonlinear Hammerstein integral equation as follows

$$
x(t)=f(t)+\int_{0}^{t} K(t, s) h(s, x(s)) d s,
$$

where the unknown function $x(t)$ takes real values.

Let $X=C([0,1])$ be the space of all real continuous functions defined on $[0,1]$. It is well known that $C([0,1])$ endowed with the metric

$$
d(x, y)=\|x-y\|=\max _{t \in[0,1]}|x(t)-y(t)| .
$$

So, $(X, d)$ is a complete metric space. Define a mapping $F: X \rightarrow X$ by

$$
F(x)(t)=f(t)+\int_{0}^{t} K(t, s) h(s, x(s)) d s, \text { for all } t \in(0,1) .
$$




\section{Assumption 3.1}

$(I)^{*} f \in C([0,1] \times(-\infty,+\infty)), f \in X$ and $K \in C([0,1] \times[0,1])$ such that $K(t, s) \geq 0$

$(I I)^{*} h(t, \cdot):(-\infty,+\infty) \rightarrow(-\infty,+\infty)$ is increasing for all $t \in(0,1)$, such that

$$
\frac{1}{2} d(x, F(x))<d(x, y)
$$

implies $|h(t, x)-h(t, y)|<\mathcal{M}(x, y)$, for all distinct $x, y \in X, t \in(0,1)$, where $\mathcal{M}(x, y)=\max \left\{|x-y|,|x-F x|,|y-F y|, \frac{|x-F y|+|y-F x|}{2}\right\} ;$

$(I I I)^{*} \max _{t, s \in[0,1]}|K v(t, s)| \leq 1$.

Theorem 3.1. Let $X=C([0,1]),(X, d), F, h, K(t, s)$ are satisfied in Assumption 3.1, then the nonlinear Hammerstein integral equation (3.1) has a unique solution $w \in C([0,1])$ and for each $x \in C([0,1])$ the iterative sequence $\left\{x_{n}=F^{n} x\right\}$ converges to the unique solution $w \in X$ of equation (3.1).

Proof. First, we show that the mapping $F: X \rightarrow X$ define by (3.2) is a Suzuki type Z-contraction. From condition $(I I)^{*}$ and $(I I I)^{*}$, for all distinct $x, y \in C([0,1])$, $t \in(0,1)$, we have

$$
\begin{aligned}
|F x(t)-F y(t)| & =\left|\int_{0}^{t} K(t, s)(h(s, x(s))-h(s, y(s))) d s\right| \\
& \leq \int_{0}^{t}|K(t, s)||h(s, x(s))-h(s, y(s))| d s \\
& \leq \int_{0}^{t}|h(s, x(s))-h(s, y(s))| d s \\
& <\int_{0}^{t} \mathcal{M}(x(s), y(s)) d s \\
& =\int_{0}^{t} \max \left\{\begin{array}{c}
|x(s)-y(s)|,|x(s)-F x(s)|,|y(s)-F y(s)|, \\
\frac{|x(s)-F y(s)|+|y(s)-F x(s)|}{2}
\end{array}\right\} d s \\
& \leq \int_{0}^{t} \max \left\{d(x, y), d(x, F x), d(y, F y), \frac{d(x, F y)+d(y, F x)}{2}\right\} d s \\
& =M(x, y) \int_{0}^{t} d s \\
& =t M(x, y) \\
& \leq M(x, y) .
\end{aligned}
$$

Hence, the mapping $F$ is a Suzuki type Z-contraction and Theorem 2.4 applies to $F$, which guarantee the existence of a unique fixed point $w \in X$. That is, $w$ is the unique solution of the nonlinear Hammerstein integral equations (3.1). For each $x \in X$, the sequence $\left\{x_{n}=F^{n} x\right\}$ converges to $w$. 


\section{COMPETING INTERESTS}

The authors declare that they have no competing interests.

\section{Authors' CONTRIBUtions}

All authors read and approved the final manuscript.

\section{ACKNOWLEDGMENTS}

This project was supported by the Theoretical and Computational Science (TaCS) Center under Computational and Applied Science for Smart Innovation Cluster (CLASSIC), Faculty of Science, KMUTT. The first author thanks for the support of Petchra Pra Jom Klao Doctoral Scholarship (Grant:21/2558) for Ph.D. student of King Mongkut's University of Technology Thonburi. The third author was supported by Rajamangala University of Technology Lanna (RMUTL) for Ph.D. program at King Mongkut's University of Technology Thonburi (KMUTT). The last author would like to thank the Thailand Research Fund (TRF) and King Mongkut's University of Technology Thonburi (KMUTT) for their joint support through the Royal Golden Jubilee Ph.D. (RGJ-PHD) Program (Grant No. PHD/0045/2555).

\section{REFERENCES}

[1] H. Argoubi, B. Samet and C. Vetro, Nonlinear contractions involving simulation functions in a metric space with a partial order, J. Nonlinear Sci. Appl. 8 (2015), 1082-1094.

[2] S. Banach, Sur les operations dans les ensembles abstraits et leur applications aux equations integrales, Fund. Math. 3 (1922), 133-181.

[3] Lj. Ćirić, N. Cakić, M. Rajović and J. S. Ume, Monotone generalized nonlinear contractions in partially ordered metric spaces, J. Fixed Point Theory Appl. 2009 (2008), Article ID 131294.

[4] F. Khojasteh, S. Shukla and S. Radenović, A new approach to the study of fixed point theorems via simulation functions, Filomat 29(6) (2015), 1189-1194.

[5] A. F. Roldan-Lapez-de-Hierro, E. Karapinar, C. Roldan-Lapez-de-Hierro and J. MartanezMorenoa, Coincidence point theorems on metric spaces via simulation functions, J. Comp. Appl. Math. 275 (2015), 345-355.

[6] P. Kumam, D. Gopal and L. Budhiyi, A new fixed point theorem under Suzuki type Z-contraction mappings, J. Math. Anal. 8(1) (2017), 113-119.

[7] X. D. Liu, S. S. Chang, Y. Xiao and L. C. Zhao, Existence of fixed points for $\Theta$-type contraction and $\Theta$-type Suzuki contraction in complete metric spaces, J. Fixed Point Theory Appl. 2016 (2016), Article ID 8.

[8] T. Suzuki, Ageneralized Banach contraction principle which characterizes metric completeness, Proc. Amer. Math. Soc. 136(5) (2008), 1861-1869.

[9] I. A. Rus, Generalized Contractions and Applications, Cluj University Press, Cluj-Napoca, 2001.

[10] I. D. Reem, S. Reich and A. J. Zaslavski, Two results in metric, fixed point theory, J. Fixed Point Theory Appl. 1 (2007), 149-157.

[11] Lj. Ćirić, B. Samet, C. Vetro and M. Abbas, Fixed point results for weak contractive mappings in ordered K-metric spaces, Fixed Point Theory 13 (2012), 59-72. 
${ }^{1}$ KMUtT-Fixed Point Theory and Applications Research Group (KMUtT-FPta), Theoretical and Computational Science Center (TaCS),

Science Laboratory Building, Faculty of Science, King Mongkut's University of Technology Thonburi (KMUTT), 126 Pracha-Uthit Road, Bang Mod, Thrung Khru, Bangkok 10140, Thailand

${ }^{2}$ KMUtTFixed Point Research Laboratory, Department of Mathematics, Room SCL 802 Fixed Point Laboratory, Science Laboratory Building, Faculty of Science, King Mongkut's University of Technology Thonburi (KMUTT), 126 Pracha-Uthit Road, Bang Mod, Thrung Khru, Bangkok 10140, Thailand E-mail address: apadcharoen@yahoo.com

E-mail address: poom.kum@kmutt.ac.th

E-mail address: splernn@gmail.com

E-mail address: parin.cha@mail.kmutt.ac.th 